\title{
Smart Home Strategies for User-Centered Functional Assessment of Older Adults
}

\author{
Vijay Kumar Ravishankar1, Winslow Burleson,*, and Diane \\ Mahoney ${ }^{3}$
}

${ }^{1}$ Arizona State University

${ }^{2}$ New York University

${ }^{3} \mathrm{MGH}$ Institute of Health Professions

(Received 14 May 2015; Accepted 18 July 2015; Published on line 1 December 2015)

*Corresponding author: wb50@nyu.edu

DOI: 10.5875 /ausmt.v5i4.952

Abstract: Successful aging, independence and capacity for aging in place involves the maintenance and preservation of individuals' physical, mental and social well-being. Elderly people need to maintain the capacity to perform both activities of daily living (ADL) and instrumental activities of daily living (IADL). Advances in Smart Home technologies are increasingly able to provide embedded assessments of an individual's functional ability in his/her home on a momentto-moment, daily, and longitudinal basis. To date, in-situ functional assessment systems and research have focused to a greater extent on the advancement of technologies rather than the multi-faceted needs and experiences of users, however the success of any technology depends more on the users than the technology itself. This paper presents strategies for user-centric approaches to identify the technical and design challenges of developing, deploying, and using functional assessment systems in homes occupied by senior citizens. Case studies involved 4 healthy older adults (aged $65+$ ), and examined the home deployment of smart home systems and interfaces aimed at assessment of a combination of ADL and IADL activities. Pre- and post-activity interviews were used to better understand issues related to desire, privacy, technological acceptance, suitability, and need fulfillment/support. The results inform strategies for usercentered functional assessment and assistive technology design and implementation, providing information capture, analysis, and delivery of in-home functional assessment that has the potential to support aging in place.

Keywords: Assistive technology, functional assessment, context aware computing, smart home, design

\section{Introduction}

Populations in both developed and developing countries are rapidly aging. It is projected that by the year 2050 , the number of people over 60 years of age will reach 2 billion [1]. Older adults generally prefer to live independently in their own homes (i.e., aging in place). However, to do so, they must be functionally stable and capable of performing essential daily activities on their own. Successful aging requires them to be aware of their cognitive and physical limitations and adapt themselves by making necessary adjustments to live independently. Even live-in caregivers of older adults who watch them perform their daily activities are not consistently able to accurately identify many subtle changes in behavior and

www.ausmt.org

Copyright (C) 2015 International Journal of Automation and Smart Technology capability [18]. Without specialized training it is hard for individuals to track these changes reliably, day in and day out. Even healthy older adults encounter challenges due to normal age-related changes. Such cognitive, physical, and perceptual changes often increase the difficulty of activities of daily living (ADLs), such as eating, bathing and dressing, and instrumental activities of daily living (IADLs), such as cooking, housework, and managing finances.

To enable older adults to age in place independently, it is critical to assess their functional ability. Sensor-based technologies have advanced substantially in recent years. The increasing popularity and availability of smart home and embedded sensor technologies present great opportunities to advance embedded assessment of functional ability. Sensors embedded into everyday objects can allow for unobtrusive, objective tracking of a 
person's activity. Analysis of the collected information can be used to assess functional ability, potentially providing sufficient awareness for more informed decisions at critical moments--a capability which is crucial to supporting and increasing individuals' capacity for independent living.

While considerable research has focused on ADL and IADL, these areas still require further investigation. Important research questions still need to be answered. What kind of data or information might be obtained by tracking ADL activities? To what extent is it possible to track ADL activities and how useful might such tracking be? Also, nearly all functional assessment systems lack metadata: They typically provide only cursory information about what happened and when, but provide little or no information describing why it happened, e.g., the reasons underlying the sensed actions. It is important for all stakeholders involved to know "why" something happened, to allow for further analysis for the development of appropriate preventive or remedial measures.

We believe that a user-centered approach to

Vijay Kumar Ravishankar, PhD, is a Research Software Engineer at Arizona State University's School of Nutrition and Health. He is responsible for developing software for improving health by providing contextual and meaningful interventions. He collaborates with various professors at the school on research and the development of innovative solutions to healthcare problems. He is responsible for the design, development, and testing of software for various projects within the school. Prior to pursuing his PhD, he worked in the software industry developing cutting edge technologies in web and mobile. His other interests include Behavior Change, Context Aware Computing, Big Data, Information Retrieval, and Mobile Computing. He earned his PhD from the School of Computing, Informatics and Decision Systems Engineering at Arizona State University.

Winslow Burleson, PhD is Associate Professor at New York University's College of Nursing, with appointments with NYU Polytechnic School of Engineering, NYU Steinhardt and NYU's Global Institute of Public Health. He earned a B.A. in Biophysics from Rice University, an M.S. in Engineering (Mechanical Engineering Product Design) from Stanford University, and his Ph.D. in Media Arts and Sciences from the MIT Media Lab. Burleson is a social inventor with a transdisciplinary program of integrated research and education focused on health and educational technologies, informatics, design, simulation, and innovation. He leads the NYU-X initiative fostering new paradigms for transdisciplinary Health, Education, Technology and Innovation across NYU's Global Network University, and beyond. He received a Google Faculty Research Award in 2013 and has been recognized by the National Academy of Engineering as "one of the nation's brightest young engineering researchers and educators."

Diane Feeney Mahoney, PhD, RN is the MGH Institute of Health Profession's School of Nursing's Jacques Mohr Research Professor and Director of Gerontechnology. She is considered one of the U.S. pioneers in the field of Gerontechnology. Her research program focuses on developing technology based interventions to help at-risk memory impaired older adults sustain their ability to remain living at home. She has numerous original research publications on using interactive technologies to educate consumers about the differences between normal forgetfulness and more serious memory loss, automated tailoring of caregivers' support and education, remote monitoring of frail elders at home from the workplace, cross cultural similarities and differences in experiences obtaining the diagnosis of dementia, dressing issues, and family caregivers impressions and responses to caregiving related technologies. She earned her PhD from the Heller School at Brandeis University in Massachusetts and is a fellow in the Gerontological Society of America and the American Academy of Nursing. advancing smart home technologies for functional assessment is fundamental to designing technologies and experiences that are well-suited for individual and caregiver needs. User-centered design approaches use a multi-stage process that incorporates end user input throughout the design process, both in developing original design assumptions, as well as in real-world tests with actual users whenever possible. Ultimately, usercentered strategies are essential to understanding "why" people are capable or incapable of living independently, and to preserving their ability to age in place. By and large, prior research has focused on technological advancements. While technological development is important, increasing the utility and efficiency of technologies requires a greater emphasis on how people actually use technology. With this motivation, our research takes a user-centered approach to designing a functional assessment system. This is particularly challenging when involving vulnerable populations such as frail older adults and those with cognitive impairments. Thus we propose an iterative approach with an initial stage that includes healthy older adults subsequently progressing to those with impairments as the technology matures.

\section{Background}

Approximately $10 \%$ of the global population has some form of disability; $20 \%$ of this population is over 70 years of age and $50 \%$ is over 85 years of age [43]. Unfortunately, these disabilities, especially when they are cognitive, are frequently overlooked by health care providers until an event, such as a fall, precipitates the presentation of disability [2]. Older adults are aware of physical deterioration before the development of overt functional limitations and respond by reducing or modifying tasks. Fried et al., 1991 [10] called this transitional functional state "preclinical disability". Researchers measure preclinical disability within a domain of tasks to identify early warning signs that disablement is in progress [10, 46, 47]. Improving capacities for the identification of preclinical disability might afford opportunities for early intervention, at a time when there may still be a greater chance to promote recovery and prevent the detrimental evolution of preclinical disability.

Clinical diagnostic practices frequently fail to identify health problems in the early stages as evaluation is often conducted after the onset of the health problem, at which time there is often little or no data about the individual's baseline functioning. Self-reported data has repeatedly been shown to be unreliable $[12,44]$ and is thus not suitable for use in clinical assessments, 
particularly once an individual's abilities have begun to decline. Clinical diagnostic practices are also limited in terms of their ecological validity as they are not performed in the individual's homes and do not account for other environmental factors. Clinical assessments performed under direct observation within the patient's home are infrequent and typically only occur after a problem arises. Such assessments can also be biased by "performance efforts" in which patients strive to act differently from how they would normally function in their everyday lives. Objective, timely, and ecologically valid information about the individual's functional abilities is important for proper diagnosis and treatment of the causes of functional decline [31]. Thus, clinicians need more frequent, less expensive, and more objective measures of an individual's functional ability with respect to their everyday activities and needs. The current research explores the potential of user-centered smart home strategies to obtain ongoing objective and costeffective functional assessments through the use of embedded technologies.

Advancement of embedded assessment occurs within the larger context of ubiquitous computing [41]. Most prior work in the field of ubiquitous computing for healthcare has been in the area of activity monitoring. Ubiquitous computing researchers have long advocated for in-home and on-body monitoring to help users assess their own health as well as that of their loved ones [32]. Sensors embedded in the home are used to collect longitudinal and contextually relevant data that can be processed to automatically detect changes in behavior patterns caused by the onset of illness. Some researchers have used sensors on mobile devices to detect patterns in everyday activities [33]. These types of systems either continuously or intermittently monitor users while they engage in a specific activity (exercise, sleep, recreation, etc.) [16]. Other studies have evaluated in-home monitoring systems with a small sample size and shorter observation period [4]. Larger, longitudinal studies correlating home and clinical assessments are generally not yet feasible for most ubiquitous computing trials. There are some exceptions, e.g., Rantz et al. [37]; and Lee et al. [20], in which in-home monitoring systems were installed for up to 18 months; however the number of participants was still small. Lee's system was designed to track only IADL activities, and did not provide compensatory strategies or real time information to assist accident/incident prevention. Another important feature absent in these systems is the ability to identify why an event, failure or departure from the standard behavior occurred in the context of functional assessment. Considering the advancements in the use of sensors to capture personal data, it is important to respect user privacy when designing embedded sensing systems. Prior literature on privacy involving older adults and technology provides some pointers on how to proceed [7, 22, 23, 30].

Functional Assessment through Activities of Daily Living

Functional assessment is a multidimensional and often interdisciplinary diagnostic process to assess and quantify an older adult's medical, psychosocial, and functional status [26]. Information gathered in this process is used by practitioners, patients, and families to develop a comprehensive plan for therapy and future care decisions, and can also help in the process of long-term care decision-making. For a person to be functionally independent, they need to be able to perform both ADL and IADL activities autonomously. Numerous studies have monitored daily living activities $[4,13,19,24,27,32,35$, $40,45]$; these systems usually collect data continuously, e.g., to monitor behavior [15], such as when a person is engaged in an exercise [38]. These systems typically indicate whether an activity was initiated or completed. However, to accurately predict or measure any decline in functional ability, it is important to not only know whether the activity was completed, but also how well the activity was performed. Embedded assessments could potentially provide information for early prediction [3] and treatment of decline and possibly further delay the onset of disability.

Many sensing systems have been developed to monitor the frequency with which various IADLs (e.g., cooking) are performed [36, 39, 40], and some of these systems correlate behaviors with clinical outcomes [17]. Some systems focus on recording the outcomes of a particular IADL such as medication taking [14]. Mihailidis et al., [28] developed a computer vision-based system to monitor the steps in a hand-washing task, to identify process errors and to provide appropriate prompts to assist the user in completing the task. Cook \& SchmitterEdgecombe (2009) [5] developed an intelligent system that can detect step errors, time lags, and missteps in IADL task processes, providing a measure of how well the task was performed. Lee (2010) [21] designed and developed a system which monitors how well individuals perform IADL activities. In addition to monitoring the frequency of task completion, Lee monitored how well the task was completed. Many researchers have attempted to develop systems to observe and monitor ADL activities. Gendron et al., 1993 [11] developed a tool to observe four daily living activities to evaluate the functional autonomy of persons suffering from dementia. Matthai et al., 2004 [36] developed a system using a probabilistic inference engine to monitor ADL activities. Their Proactive Activity (PROACT) toolkit represents activities as a probabilistic sequence of objects and develops a model to identify patterns of usage. Fluery et al., [9] designed an apartment embedded with 
sensors to classify daily living activities through the use of support vector machines. The data from these sensors were collected and analyzed for the early detection of loss of autonomy. Some research projects are working towards ADL task assistance such as that developed by Peters et al., 2014 for brushing teeth [34].

\section{Embedded Assessment Architecture}

The proposed system for the functional homebased assessment of ADL and IADL activities is based on the Game As Life, Life As Game (GALLAG) framework [5] (Fig. 1), a collection of software and hardware technologies for seamless interfacing a diverse array of smart home and consumer electronic devices. The GALLAG framework provides a basic infrastructure necessary to build complex embedded systems. The following is a description of two ADL activities (dressing and brushing teeth) and two IADL activities (coffee making and medication taking), which we developed and employed in the homes of healthy older adults. A skin conductance sensor, a custom version of Affectiva's $Q$ sensor, was also used to detect subjects' levels of arousal (an indicator of emotional state) [6].

\section{Intelligent Dresser}

To monitor dressing, a customized dresser was developed (Fig. 2). The design of this intelligent dresser followed a user centered design process. Focus groups and interviews were conducted with caregivers to identify the most efficient and usable design for older adults. The 4-drawer dresser is a standard consumer dresser, fitted with sensors to track dressing activity. Each drawer is fitted with an $\times 10$ door sensor to detect the drawer's open/closed state. Each clothing item can be placed in separate drawers. To minimize the complexity associated with tracking a dressing activity, only shirts and pants are tracked. Two ps3eye cameras are used to track fiducial markers attached to the clothing items to identify garment orientation and location. One camera is placed on top of the dresser, while the other one is placed on the front panel of the middle drawer. The data from all devices is wirelessly sent to the GALLAG central server, also placed in the user's home.

\section{Smart Coffee Tracker}

Coffee making is a common sequential daily task. To monitor various steps of making coffee, we embedded small, unobtrusive sensors in the coffee machine and on the cabinets containing the coffee filters and ground coffee (Fig. 3). The sensors in the cabinets track whether the user opened the cabinets to add the coffee filter and ground coffee. The sensors in the coffee machine determine whether the filter basket is opened, whether water is added to the machine, whether the switch was turned on, and the temperature of the carafe. All the sensors transmit data wirelessly to the server. Based on the data received, the system can identify which steps were performed, how long it took to perform these steps and whether any errors were committed during execution. While the system is capable of providing supportive messages to prompt error rectification, the use of voice prompts was beyond the scope of our case study and the current research.

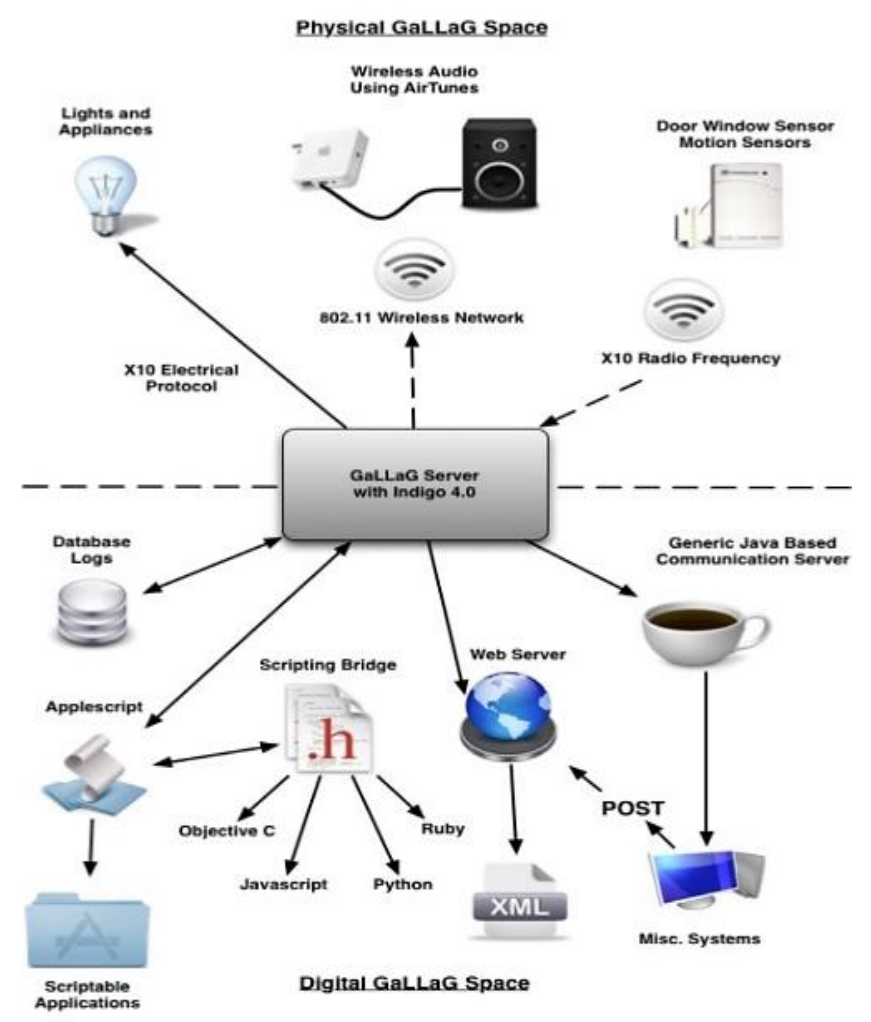

Figure 1. GALLAG Framework.

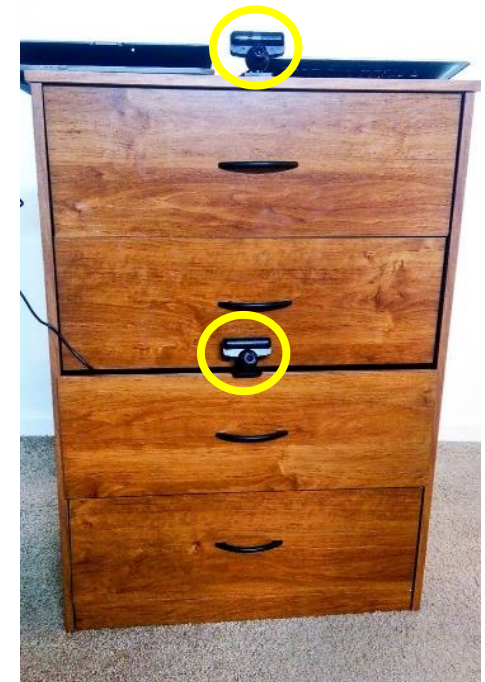

Figure 2. Intelligent Dresser with two ps3 eye cameras. 


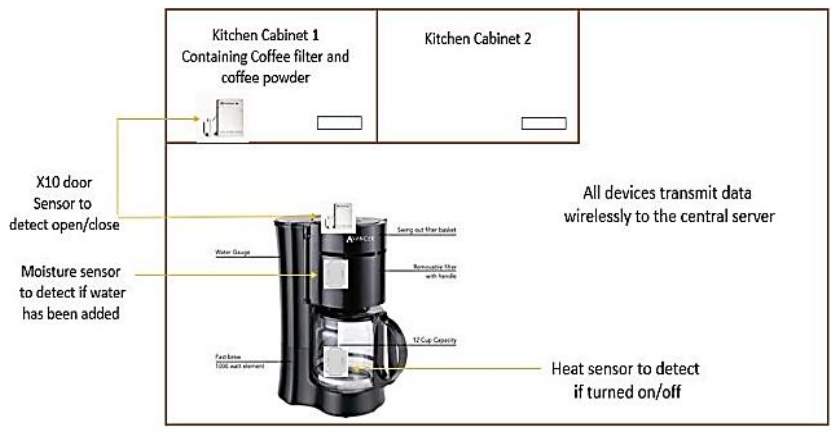

Figure 3. Smart Coffee Tracker.

\section{Smart pillbox}

To track medication taking, a smart pillbox (Fig. 4) was devised. While the current design could be improved upon in terms of efficiency, none of the case study participants had any difficulty using it. It is a standard pillbox augmented with sensors to detect whether the user opened or closed a particular cell. The system can detect whether the user opened the wrong cell based on their daily routine. The system can be customized to monitor different medication-taking habits (e.g., pouring a glass of water first or organizing pills first. Based on the data received, the system can identify errors and the time taken to perform each task. As mentioned above, although not part of this case study, voice guidance can also be provided to support participants in the successful completion of the activity.

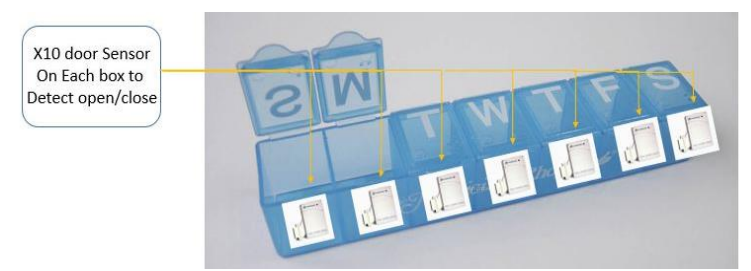

Figure 4. Smart Pillbox.

\section{Smart Toothbrush}

Several designs were considered in developing a smart toothbrush. The initial design attached a sensor to the toothbrush, but users found it difficult to hold and brush normally. To track brushing activity during the study, a motion sensor was attached to a standard toothbrush (Fig. 5) using a string. Tooth brushing habits vary across individuals in terms of duration and intensity, thus customizing software to accurately detect brushing activity and changes in activity is important. Based on the data received, the system can identify when and for how long a participant performed the task.

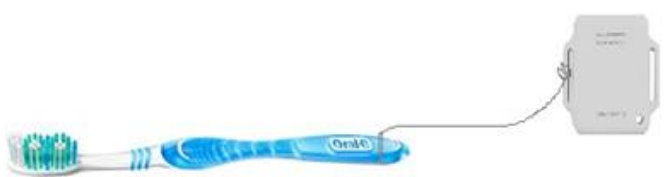

Figure 5. Smart Toothbrush.

\section{System Components}

As shown in Fig. 6, the system features three key elements, (a) sensing, (b) processing, and (c) presenting essential to the assessment of an individual's functional abilities. Sensing is responsible for sensing events and capturing relevant information necessary for analysis through multiple sensors embedded within daily use objects. As raw data is captured it is stored on the system server.

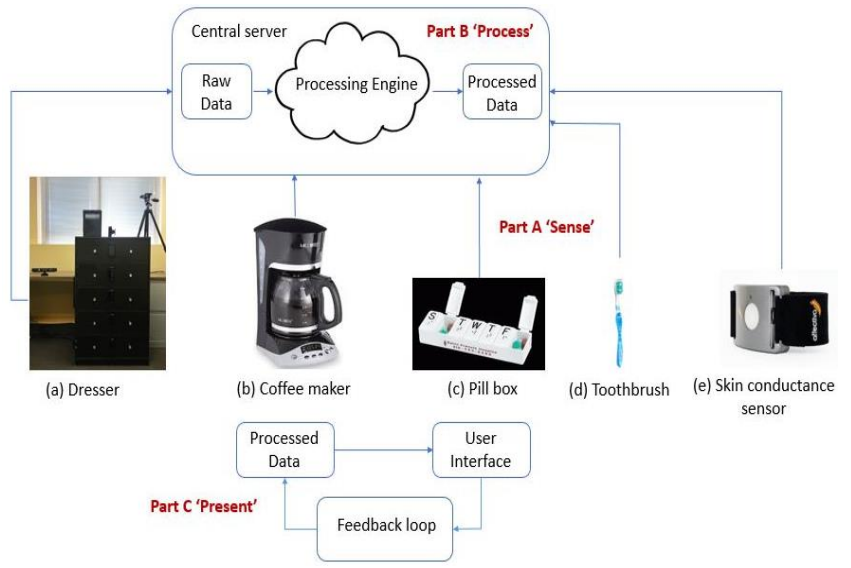

Figure 6. System Components.

The process component is responsible for sifting through large amounts of data to identify relevant and meaningful information, storing the results in a MySQL Database. The present component is responsible for enabling participants to access and make sense of the captured data. It extracts relevant information from the database and presents it to the user in an easy to understand format. The design of the user interface (Fig. 7) follows user-centered design principles proposed by Story and Molly [44]. It also follows an adaptable design principle allowing it to adapt to the data at hand, never showing more than is immediately necessary. For example: The feedback form used to gather near-ground truth and to eliminate false positives is presented only if the system finds an anomaly. If there is no anomaly, the form is hidden from the user.

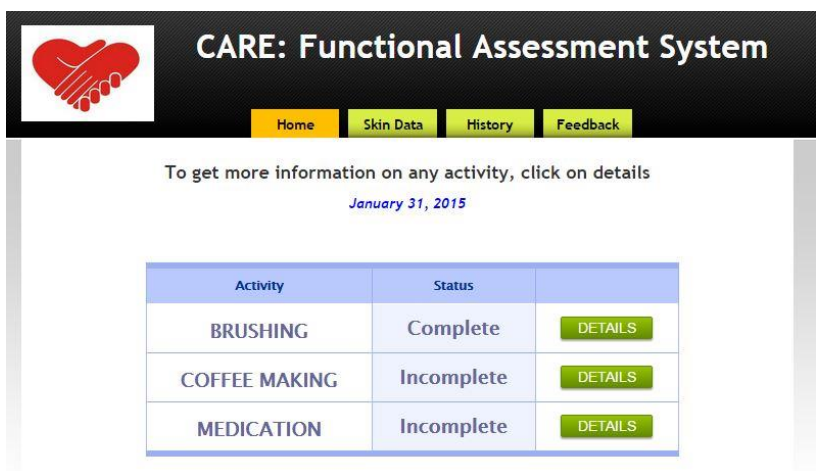

Figure 7. User Interface (home screen). 


\section{Methodology}

The current research focuses on identifying technical and design challenges involved in the deployment and use of a functional assessment system in the home environments of older adults. Even relatively healthy adults without disabilities begin to experience limitations in living independently, encountering challenges due to normal age-related changes. Typical age-related changes (e.g., cognitive, physical, perceptual) can make ADLs and IADLs increasingly difficult.

We conducted in-home case studies with 4 healthy older adults ( 2 male and 2 female) over a period of two weeks. All participants were aged 65 and over (average age was 68). Three participants were living independently, while the fourth was sharing her home with her sister. Three of the 4 participants were living in 2-bedroom apartments while the fourth participant was living in a 1bedroom apartment.

Case study participants were asked to use our prototype system to perform four prescribed daily activities for a period of two weeks. None of the participants had been diagnosed with any cognitive or physical disabilities and all consented to participate. The study protocol received human subject testing approval from the Arizona State University (ASU) Internal Review Board (IRB) [STUDY00002063].

To assess suitability for case studies participation, candidates were selected based on a brief questionnaire covering their age, living situation, care requirements, and cognitive and physical disabilities. The case study was then conducted in three different phases. In the first phase, selected participants were asked to take standardized physical (Timed Up and Go, TUG [34]) and cognitive (Mini Mental State Examination, MMSE [8]) tests to further ensure they had no disability which could prevent them from performing the required daily activities. The second phase included system installation, training on how to access data, and an audio-recorded pre-study interview. The third phase included use of the embedded functional assessment technologies in the participant's home and concluded with an audio-recorded post-study interview. Two in-person interviews were conducted at each participant's home (once before the start of the study and once after the study was complete). Each interview lasted 30-60 minutes and was standardized across participants, audio recorded, and transcribed. The interviews were subjected to inductive analysis [46]. Interview data was analyzed and grouped into categories using five key features: category label, category description, text associated with category, links, and the type of model in which the category is embedded. Inductive analysis provided a convenient and efficient way of analyzing the qualitative data and was used to uncover key themes that help inform a potentially generalizable model.

Participants were encouraged to perform the required activities naturally, on their own schedule. Care was taken to ensure the system would not interfere with their daily routine. Passive system monitoring tracked their daily activities and logged data to the local server. Participants were given the option to withdraw from the study at any point for any reason.

\section{Results and Analysis}

Everyday home life presents unique challenges for elderly people, and aging at home requires ongoing efforts by designers, technologists and healthcare providers to collaborate to develop effective and innovative solutions.

From a sensor system perspective, the two challenges most frequently encountered among the four participants in our case study were (a) sensors losing connection with receivers and (b) sensors unable to communicate (either due to sensor malfunction or battery life), rendering them useless. For the first situation (a), if the sensor was reset and re-connected to the receiver, the problem was solved. However, for the second scenario (b), the non-responsive sensor had to be replaced. Among the four participants, two sensors (a motion sensor and moisture sensor) were replaced, but the source of the failure in these two devices remains undetermined (the participants were not aware of the problem until the researcher informed them that the devices were not collecting data). In addition, environmental issues raised other challenges. Router settings in participant 1's home prevented the REST service (the internet architecture parameter customized to enable data logging to a server) from calling the custom URL, thereby preventing it from logging the data. In participant 3's home, the wireless network was disconnected several times, preventing data logging. In the homes of participants 2 and 3, the $x 10$ sensors were moved from their original positions (the sensor which was attached to the coffee maker lid was detached and the participants did not notice for several days) preventing the system from detecting user actions. In each of the above scenarios, the participants were not aware of the problem. With the exception of the skin conductance sensor with limited battery life, no sensors experienced power loss and the internal batteries lasted for the duration of the study.

To remedy these sensor issues, we recommend an in-home functional assessment system to monitor the status of the sensors within different objects, identify any problems that arise and fix them at the earliest opportunity, without involving the user. The process of 
identifying and rectifying errors should be automated and occur either by the researcher intervention or automatically by the system. Prior research shows that expecting caregivers or the care recipients to perform additional tasks is not a viable approach [27].

With respect to the different activities investigated in the case studies, medication tracking was by far the best received and performed most accurately. The coffee making and teeth brushing sensors were not $100 \%$ reliable but performed well overall. Dressing was used by only one of the participant and the skin conductance sensor's battery only lasted for 2 days in these trials.

Participants were reluctant to perform dressing activity because of the use of cameras on the dresser, which were perceived as impinging on participant privacy. Participant 2 (male) was initially willing to perform the dressing activity, but later changed his mind, citing an unexpected feeling of having his privacy violated by the cameras. Upon his request, the cameras were removed and dressing was removed from the list of activities being tracked for his participation.

This experience with the deployment of the dressing system is an important finding because it shows how perception and emotional response to various activities can change over time. The participant was well versed with technology, has experience building assistive technology for the disabled and was comfortable with being recorded on video. However, he was surprised by his change of heart, indicating that extra care must be taken in designing technology for older adults considering their experience with technology, education, and personality.

Another finding from the case studies was that not all participants used the user interface to monitor their activities. The user interface (Fig. 7) provided the following data about the three activities; daily occurrence; error rate and type (if any); and skin conductance. All participants reported they believed performance monitoring would not provide any new or important information. While all agreed that activity tracking is important, they did not currently feel the need to monitor activity performance and would not do it on a daily basis.

This is interesting considering that our initial hypothesis was that the data provided would help to improve awareness and participant feedback confounded our expectations. Participants instead asked for real time feedback through audio prompts if the system found something out of the ordinary (an anomaly). This provided valuable information regarding the manner in which information is captured and presented to the user. Information delivery is an important aspect of any functional assessment system; if information is not presented at the right time and in the right way, then system goals can not be met.
With respect to the skin conductance sensor and its ability to capture indicators of emotional state as users engage in ADL and IADL activities, our interest was to compare performance on activities with arousal states. However, short battery life in the skin conductance sensor (under 2 days) limited their value. The software used to capture skin conductance values ran continuously, 24 hours a day, and the continuous communication with the server can quickly drained the sensor battery. The limited data prevented analysis of the participant's arousal levels during the activities. We feel that collection of arousal levels has potential value in informing future research and design of interventions. We recommend that future research focus on reducing power intensity and communication requirements to increase sensor usability to permit integration of this promising technology.

When asked about striking a balance between the potential for the proposed system to allow for independent living against privacy concerns, all but one participant preferred independence over privacy. The two male participants did not like the idea of sharing the information captured about their functional ability with their loved ones, while the two female participants wanted their family to know if there was a problem so they could help. Male and female users may differ in terms of their perception of the privacy/independence tradeoff, and further research is warranted with respect to the design of assistive devices for elderly populations.

Analysis of the data collected from each of the four participants revealed several common themes. In particular, the case study and interview results emphasized the importance of focusing on user experience, catering to independence and/or privacy needs and adapting or customizing technology to the user's individual qualities. Participants unanimously agreed that there is a need for this kind of technology and that this could help them perform daily activities independently while reducing the burden on caregivers. Likewise, such technologies need to be reliable and robust over time, raising the need for software protocols that can identify a sensor or data failure and quickly fix it without user intervention.

\section{Limitations}

The case study involved only 4 individuals for a 2 week period, and this study is subject to several limitations that must be acknowledged:

- The captured data should not be inappropriately extrapolated to characterize the general elderly population. In addition, cognitive and physical impairment symptoms can vary significantly from individual to 
individual, and certain forms of cognitive impairment overlap. In future studies, researchers should consider recruiting greater numbers of participants with a broad range of physical and cognitive impairments for in-home assessments.

- $\quad$ Functional assessment based on embedded sensors depends largely on sensor efficiency. Sensors may interpret changes in user behavior as anomalies where they may in fact simply be normal variability in otherwise daily routine.

- The sensors and other devices used for this study rely on uninterrupted power supply and Internet connectivity. Intermittent communication could cause data loss and affect analysis results.

- Data collected through embedded sensors should be considered only a single source of information. Observed behavior requires further and more holistic explanations in the specific context in which it was collected. To provide better insight into the data, we included a feedback mechanism to capture user information. When the system finds an anomaly, a questionnaire is sent to the user to record their personal perspective of the event. Since users did not use the interface provided to them, they did not record their perspective, which would have provided valuable information to help establish ground truth.

\section{Conclusion and Future Work}

The findings from this study indicate the importance of focusing on user-centered design and customizing technologies according to the personal needs of users. Results suggest that the proposed system can reliably track dressing activity, but additional testing is needed before long-term deployment. Sensor battery life may require periodic monitoring, or the sensors can be redesigned to allow for periodic recharging to reduce data loss. Future evaluations of functional assessment systems might include more metrics to maximize system efficiency and reliability. Interview results indicate the need for guidance using audio prompts. While the current system can provide audio prompt guidance it has not yet been deployed for in-home use. Future studies should investigate this functionality for potential benefit of both older adults and caregivers. Future work could also assess other activities such as sleep, physical activity, and social interaction. As monitoring technologies become more affordable, scalable, and interoperable, the development of new devices will provide opportunities to track additional activity types. The findings from this study demonstrate the critical importance of involving older adults and their caregivers to determine their engagement preferences when designing systems with multiple monitoring options.

\section{References}

[1] US Department of Health and Human Services, "Administration on Aging (AOA). Administration for Community Living. Profile of older Americans," 2017.

[2] S. J. Andersen and M. A. Johnson, "Caring for patients on the edge of decline," the American Journal of Nursing, vol. 96, no. 12, pp. 16B-16D, 1996.

doi: $10.2307 / 3465112$

[3] P. Barberger-Gateau, C. Fabrigoule, C., Helmer, I. Rouch, and J. Dartigues, "Functional impairment in instrumental activities of daily living: An early clinical sign of dementia?" Journal of American Geriatrics Society, vol. 47, no. 4, pp. 456-462, 1999. doi: 10.1111/j.1532-5415.1999.tb07239.x

[4] T. S. Barger, D. E. Brown and M. Alwan, "Healthstatus monitoring through analysis of behavioral patterns," in proceedings of IEEE Transactions on Systems, Man, and Cybernetics - Part A: Systems and Humans, Jan. 2005, vol. 35, no. 1, pp. 22-27.

doi: 10.1109/TSMCA.2004.838474

[5] W. Burleson, N. Newman, and R. Brotman, "Empowering independent living for people with autism: Designing supportive, low-cost, interactive e-health environments," in proceedings of the 7th International Conference on Persuasive Technology: Design for Health and Safety, Linköping, Sweden, June 6-8, 2012, pp.13-30. doi: $10.1007 / 978-3-642-31037-92$

[6] W. Burleson and R. W. Picard, "Gender-Specific Approaches to Developing Emotionally Intelligent Learning Companions," IEEE Intelligent Systems, vol. 22, no. 4, pp. $62-69,2007$. doi: $10.1109 /$ MIS.2007.69

[7] K. E. Caine, K. E. Burnham, A. D. Fisk, and W. A. Rogers, "Privacy concerns and disclosure behavior in a health setting," in proceedings of the Human Factors and Ergonomics Society 52nd Annual Meeting, Santa Monica, CA, 2008, pp. 1785-1789. doi: $10.1177 / 154193120805202201$

[8] J. R. Cockrell and M. F. Folstein, "Mini-mental state examination," Principles and practice of geriatric psychiatry, pp.140-141, 2002. doi: $10.1002 / 0470846410 . c h 24$

[9] D. Ding, R. A. Cooper, P. F. Pasquina, and L. FiciPasquina, "Sensor technology for smart homes," Maturitas, vol. 69, no. 2, pp. 131-136, 2011. doi: 10.1016/j.maturitas.2011.03.016

[10] L. Fratiglioni, S. Paillard-Borg, and B. Winblad, "An active and socially integrated lifestyle in late life 
might protect against dementia," The Lancet Neurology, vol. 3, no. 6, pp. 343-353, 2004. doi: $10.1016 /$ S1474-4422(04)00767-7

[11] L. P. Fried, K. Bandeen-Roche, P. H. Chaves, and B. A. Johnson, "Preclinical mobility disability predicts incident mobility disability in older women," The Journals of Gerontology Series A: Biological Sciences and Medical Sciences, vol. 55, no. 1, pp. M43-M52, 2000.

doi: 10.1093/gerona/55.1.M43

[12] L. P. Fried, S. J. Herdman, K. E. Kuhn, G. Rubin, and K. Turano, "Preclinical disability: Hypotheses about the bottom of the iceberg," Journal of Aging and Health, vol. 3, no. 2, pp. 285-300, 1991. doi: $10.1177 / 089826439100300210$

[13] A. A. Gorin and A.A. Stone, "Recall biases and cognitive errors in retrospective self-reports: A call for momentary assessments," Handbook of Health Psychology, Hove, UK: Psychology Press, 2001, pp. 405-413. doi: 10.1016/B978-012402771-8/50003-9

[14] J. M. Guralnik, L. Ferrucci, E. M. Simonsick, M. E. Salive, and R. B. Wallace, "Lower-extremity function in persons over the age of 70 years as a predictor of subsequent disability," New England Journal of Medicine, vol. 332, no. 9, pp. 556-562, 1995. doi: 10.1056/NEJM199503023320902

[15] S. S. Intille, "Ubiquitous Computing Technology for Just-in-Time Motivation of Behavior Change," Medinfo, vol. 107, pp. 1434-1437, 2004. doi: $10.1007 /$ s00779-010-0299-3

[16] S. S. Intille, "A new research challenge: Persuasive technology to motivate healthy aging. Information Technology in Biomedicine," in IEEE Transactions on Information Technology in Biomedicine, vol. 8, no. 3, pp. 235-237, 2004. doi: 10.1109/TITB.2004.835531

[17] J. Jimison, M. Pavel, J. Pavel, and J. McKanna, "Home monitoring of computer interactions for the early detection of dementia. Engineering in Medicine and Biology Society," in proceedings of Engineering in Medicine and Biology Society, 2004. IEMBS'04. 26th Annual International Conference of the IEEE, 2004, vol. 2, pp. 4533-4536. doi: 10.1109/IEMBS.2004.1404258

[18] A. Kapoor, W. Burleson, and R. W. Picard, "Automatic prediction of frustration," International Journal of Human-Computer Studies, vol. 65, no. 8, pp. 724736, 2007. doi: $10.1016 /$ ij.ijhcs.2007.02.003

[19] N. Kemp, H. Brodaty, D. Pond, and G. Luscombe, "Diagnosing Dementia in Primary Care: The Accuracy of Informant Reports," Alzheimer Disease
\& Associated Disorders, vol. 16, pp. 171-176, 2002. doi: 10.1097/00002093-200207000-00007

[20] J. A. Kientz, S. N. Patel, B. Jones, E. Price, E. D. Mynatt, and G. D. Abowd, "The georgia tech aware home," in proceeding of $\mathrm{CHI}$ '08 Extended Abstracts on Human Factors in Computing Systems, Florence, Italy, April 5-10, 2008, pp.3675-3680.

doi: $10.1145 / 1358628.1358911$

[21] I. Korhonen, P. Paavilainen, and A. Särelä, "Application of ubiquitous computing technologies for support of independent living of the elderly in real life settings," in proceedings of UbiHealth 2003: The 2nd International Workshop on Ubiquitous Computing for Pervasive Healthcare Applications, 2003, p. 2. doi: 10.1109/MPRV.2003.1228521

[22] M. Kwasny, K. E. Caine, W. A. Rogers, and A. D. Fisk, "Privacy and technology: folk definitions and perspectives," in proceeding of $\mathrm{CHI}$ Conference, ACM, 2008, pp. 3291-3296.

doi: $10.1145 / 170791.170848$

[23] L. Lorenzen-Huber, M. Boutain, L. J. Camp, K. Shankar, and K. H. Connelly, "Privacy, technology, and aging: A proposed framework," Ageing International, vol. 36, no. 2, pp. 232-252, 2011. doi: 10.1007/s12126-010-9083-y

[24] D. F. Mahoney, W. Burleson, C. Lozano, V. Ravishankar, and E. Mahoney, "Development of a responsive emotive sensing system (DRESS) to aid persons with dementia dress independently," Gerontechnology, vol. 13, no. 2, p. 259, 2014. doi:10.4017/gt.2014.13.02.020.00

[25] D. F. Mahoney, W. Burleson, and E. Mahoney, "Context aware automated prompting for dressing: Concept feasibility," Gerontechnology, vol. 11, no. 2, p. 298, 2012. doi: $10.4017 /$ gt.2012.11.02.281.00

[26] D. F. Mahoney, P. Mutshler, B. Tarlow, and E. Liss, "Real world implementation lessons and outcomes from the Worker Interactive Networking (WIN) project: workplace-based online caregiver support and remote monitoring of elders at home," Telemedicine and e-health, vol. 14, no. 3, pp. 224234, 2008. doi: $10.1089 /$ tmj.2007.0046

[27] D. F. Mahoney, R. B. Purtilo, F. M. Webbe, M., Alwan, A. J. Bharucha, T. D. Adlam, and S. A. Becker, "Inhome monitoring of persons with dementia: Ethical guidelines for technology research and development," Alzheimer's and Dementia, vol. 3, no. 3, pp. 217-226, 2007. doi: $10.1016 /$ i.jalz.2007.04.388

[28] K. Matsouoka, "Smart house understanding human 
behaviors: Who did what, where, and when," in proceeding of the 8th World Multi-Conference on Systems, Cybernetics, Orlando, FL, July 18-21, 2004, pp. 181-185.

doi: $10.1177 / 0956474817730763$

[29] M. Matteson, "Functional assessment of the elderly," Nurse Practitioner Forum, vol. 8, no. 3, p. 91, 1997. doi: $10.1097 / 00006205-198308020-00010$

[30] A. S. Melenhorst, W. A. Rogers, and D. G. Bouwhuis, "Older adults' motivated choice for technological innovation: Evidence for benefit-driven selectivity," Psychology and Aging, vol. 21, pp. 190-195, 2006. doi: $10.1037 / 0882-7974.21 .1 .190$

[31] A. Mihailidis, B. Carmichael, J. Boger, J., and G. Fernie, "An intelligent environment to support aging-in-place, safety, and independence of older adults with dementia, " in proceeding of UbiHealth 2003: The 2nd International Workshop on Ubiquitous Computing for Pervasive Healthcare Applications, 2003. doi: $10.5220 / 0002572801010110$

[32] M. Morris, J. Lundell, and E. Dishman, "Ubiquitous computing for mild cognitive impairment: A prototype for embedded assessment and rehearsal," Gerontologist, vol. 43, pp. 393-394, 2003. doi: 10.1093/geront/gnv519.03

[33] E. Mynatt and W. Rogers, "Developing technology to support the functional independence of older adults," Ageing International, vol. 27, no. 1, pp. 2441, 2001. doi: $10.1007 / \mathrm{s} 12126-001-1014-5$

[34] nTAG. (2004). nTag interactive.

[35] O. Okonkwo, R. Griffith, D. Vance, D. Marson, K. Ball, and V. Wadley, "Awareness of Functional Difficulties in Mild Cognitive Impairment: A Multidomain Assessment Approach," J Am Geriatr Soc, vol.57, pp. 978-84, 2009 doi: 10.1111/j.1532-5415.2009.02261.x

[36] C. Owsley, M. Sloane, G. McGwin Jr, and K. Ball, "Timed instrumental activities of daily living tasks: Relationship to cognitive function and everyday performance assessments in older adults," Gerontology, vol. 48, no. 4, pp. 254-265, 2000. doi: $10.1159 / 000058360$

[37] M. Philipose, K. P. Fishkin, D. Fox, H. Kautz, D. Patterson, and M. Perkowitz, "Guide: Towards understanding daily life via auto-identification and statistical analysis," in proceeding of the Int. Workshop on Ubiquitous Computing for Pervasive Healthcare Applications (Ubihealth), 2003. doi: 10.1109/MPRV.2005.7

[38] D. Podsiadlo, and S. Richardson, "The timed" Up \& Go": a test of basic functional mobility for frail elderly persons," Journal of the American geriatrics Society, vol. 39, no. 2, pp. 142-148, 1991. doi: 10.1111/i.1532-5415.1991.tb01616.x

[39] M. E. Pollack, L. Brown, D. Colbry, C. E. McCarthy, C. Orosz, B. Peintner, and I. Tsamardinos, "Autominder: An intelligent cognitive orthotic system for people with memory impairment," Robotics and Autonomous Systems, vol. 44, no.3-4, pp. 273-282, 2003.

doi: $10.1016 /$ S0921-8890(03)00077-0

[40] M. Rantz, S. D. Scott, S. J. Miller, M. Skubic, L. Phillips, G. Alexander, R. J. Koopman, K. Musterman, and J. Back, "Evaluation of Health Alerts from an Early Illness Warning System in Independent Living," Comput Inform Nurs, vol. 31, no. 6, pp. 274-280, 2013.

doi:10.1097/NXN.0b013e318296298f

[41] L. Rosenberg, A. Kottorp, and L. Nygård, "Readiness for Technology Use With People With Dementia The Perspectives of Significant Others," Journal of Applied Gerontology, vol. 31, no. 4, pp. 510-530, 2012.

doi:10.1177/0733464810396873

[42] M. Stikic, T. Huynh, K. Van Laerhoven, and B. Schiele, "ADL recognition based on the combination of RFID and accelerometer sensing," in proceedings of Pervasive Computing Technologies for Healthcare, 2008, pp.258-263.

doi: 10.1109/PCTHEALTH.2008.4571084.

[43] E. Stone and M. Skubic, "Fall Detection in Homes of Older Adults Using the Microsoft Kinect," IEEE Journal of Biomedical and Health Informatics, vol. 19, no. 1, pp. 290-301, 2014

doi: $10.1109 / \mathrm{JBHI} .2014 .2312180$

[44] M. F. Story, "Maximizing usability: The principles of universal design," Assistive Technology, vol. 10, no. 1, pp. 4-12, 1998. doi: $10.1080 / 10400435.1998 .10131955$

[45] E. M. Tapia, S. S. Intille, and K. Larson, "Activity recognition in the home using simple and ubiquitous sensors," in proceeding of International conference on pervasive computing, 21-23 April, 2004, Linz and Vienna, Austria, pp.158-175. doi: 10.1007/978-3-540-24646-6 10

[46] D. R. Thomas, "A general inductive approach for analyzing qualitative evaluation data," American Journal of Evaluation, vol. 27, no. 2, pp. 237-246, 2006.

doi: $10.1177 / 1098214005283748$

[47] M. Weiser, "The Computer for the 21st Century," Scientific American, vol. 265, no. 3, pp. 94-105, 1991. doi: 10.1038/scientificamerican0991-94 